\title{
(6) OPEN ACCESS \\ Clinical benefit of 1-year certolizumab pegol (CZP) add-on therapy to methotrexate treatment in patients with early rheumatoid arthritis was observed following CZP discontinuation: 2-year results of the C-OPERA study, a phase III randomised trial
}

\author{
Tatsuya Atsumi, ${ }^{1}$ Yoshiya Tanaka, ${ }^{2}$ Kazuhiko Yamamoto, ${ }^{3}$ Tsutomu Takeuchi, \\ Hisashi Yamanaka, ${ }^{5}$ Naoki Ishiguro, ${ }^{6}$ Katsumi Eguchi, ${ }^{7}$ Akira Watanabe, ${ }^{8}$ \\ Hideki Origasa, ${ }^{9}$ Shinsuke Yasuda, ${ }^{1}$ Yuji Yamanishi, ${ }^{10}$ Yasuhiko Kita, ${ }^{11}$ \\ Tsukasa Matsubara, ${ }^{12}$ Masahiro Iwamoto, ${ }^{13}$ Toshiharu Shoji, ${ }^{14}$ Osamu Togo, ${ }^{14}$ \\ Toshiyuki Okada, ${ }^{15}$ Désirée van der Heijde, ${ }^{16}$ Nobuyuki Miyasaka, ${ }^{17}$ Takao Koike ${ }^{1,18}$
}

Handling editor Tore K Kvien

- Additional material is published online only. To view please visit the journal online (http://dx.doi.org/10.1136/ annrheumdis-2016-210246).

For numbered affiliations see end of article.

\section{Correspondence to} Professor Tatsuya Atsumi, Division of Rheumatology, Endocrinology and Nephrology, Graduate School of Medicine, Hokkaido University, North 15 , West 7, Kita-ku, Sapporo, Hokkaido 060-8638, Japan; at3tat@med.hokudai.ac.jp

Received 20 July 2016 Revised 7 December 2016 Accepted 1 January 2017 Published Online First 2 February 2017

CrossMark

To cite: Atsumi T, Tanaka Y, Yamamoto $\mathrm{K}$, et al.

Ann Rheum Dis

2017:76:1348-1356.

\section{ABSTRACT}

Objectives To investigate the clinical impact of 1-year certolizumab pegol (CZP) therapy added to the first year of 2-year methotrexate (MTX) therapy, compared with

2-year therapy with MTX alone.

Methods MTX-naïve patients with early rheumatoid arthritis (RA) with poor prognostic factors were eligible to enter Certolizumab-Optimal Prevention of joint damage for Early RA (C-OPERA), a multicentre, randomised, controlled study, which consisted of a 52week double-blind (DB) period and subsequent 52-week post treatment (PT) period. Patients were randomised to optimised MTX+CZP $(n=159)$ or optimised MTX +placebo (PBO; $n=157)$. Following the DB period, patients entered the PT period, receiving MTX alone $(\mathrm{CZP}+\mathrm{MTX} \rightarrow \mathrm{MTX} ; \mathrm{n}=108, \mathrm{PBO}+\mathrm{MTX} \rightarrow \mathrm{MTX} ; \mathrm{n}=71)$. Patients who flared could receive rescue treatment with open-label CZP.

Results 34 CZP+MTX $\rightarrow$ MTX patients and 14 PBO + MTX $\rightarrow$ MTX patients discontinued during the PT period. From week 52 through week 104, significant inhibition of total modified total Sharp score progression was observed for CZP+MTX versus PBO +MTX (week 104: $84.2 \%$ vs $67.5 \%(p<0.001)$ ). Remission rates decreased after CZP discontinuation; however, higher rates were maintained through week 104 in $\mathrm{CZP}+\mathrm{MTX} \rightarrow \mathrm{MTX}$ versus $\mathrm{PBO}+\mathrm{MTX} \rightarrow \mathrm{MTX}$ ( $41.5 \%$ vs $29.3 \%$ ( $p=0.026), 34.6 \%$ vs $24.2 \%$ $(p=0.049)$ and $41.5 \%$ vs $33.1 \%(p=0.132)$ at week 104 in SDAI, Boolean and DAS28(erythrocyte sedimentation rate) remission. CZP retreated patients due to flare $(n=28)$ showed rapid clinical improvement. The incidence of overall adverse events was similar between groups.

Conclusions In MTX-naïve patients with early RA with poor prognostic factors, an initial 1 year of add-on CZP to 2-year optimised MTX therapy brings radiographic and clinical benefit through 2 years, even after stopping CZP.

Trial registration number NCT01451203.

\section{INTRODUCTION}

Rheumatoid arthritis (RA) is a chronic inflammatory disease characterised by progressive inflammatory synovitis. This results in the destruction of articular cartilage and marginal bone, which is generally thought to be irreversible. ${ }^{1}$ Recent studies have demonstrated that the early treatment of patients with antirheumatic drugs is associated with a reduction in inflammation, greater inhibition of structural damage and better long-term outcomes. ${ }^{2}{ }^{3}$ Furthermore, early aggressive treatment with biological disease-modifying antirheumatic drugs (bDMARDs), such as antitumour necrosis factors (TNFs), was reported to be highly effective at reducing disease progression. ${ }^{4}$ The effect of treatment discontinuation/tapering following successful inhibition of disease progression as a result of using bDMARDs early in the course of the disease has yet to be fully investigated; however, there is the possibility that the positive disease trajectory may be maintained following treatment cessation.

Certolizumab pegol (CZP) is a humanised anti-TNF antibody fragment conjugated to polyethylene glycol, approved for the treatment of inflammatory diseases, including RA. The efficacy and safety of CZP in combination with methotrexate (MTX) during the early stages of RA was assessed in the Certolizumab-Optimal Prevention of joint damage for Early RA (C-OPERA) study. This study consisted of two periods: a 52-week double-blind (DB) period during which patients received either $\mathrm{CZP}$ or placebo $(\mathrm{PBO})$ together with MTX, and a subsequent 52-week post-PBO/ CZP treatment (PT) period in which patients received MTX therapy without CZP or PBO. Results from the DB period, which showed significant inhibition of structural damage and a reduction in the severity of RA symptoms following treatment with $\mathrm{CZP}+\mathrm{MTX}$ compared with $\mathrm{PBO}$ + MTX, have been reported. ${ }^{4}$ Here, we report the 
2-year overall results including the PT period, which investigated whether the clinical benefits of initial 1-year CZP+MTX therapy were sustained through a subsequent 1-year period where patients received MTX alone.

\section{METHODS}

\section{Patients}

The eligibility criteria for the DB period were previously reported. ${ }^{4}$ Brief details are provided in the online supplementary materials.

\section{Study design}

C-OPERA (NCT01451203) was a multicentre, DB, PBO-controlled, randomised, parallel-group study conducted in Japan and was previously described. ${ }^{4}$ Full details of the study design are provided in the online supplementary materials.

\section{Efficacy assessments}

Efficacy assessments were performed every 8 weeks during the PT period. The primary analysis of the PT period was change in van der Heijde modified total Sharp score (mTSS) from baseline at week 104, which compared the progression of joint damage between the CZP + MTX $\rightarrow$ MTX and PBO + MTX $\rightarrow$ MTX groups. Inhibition of joint damage progression was assessed using mTSS at weeks 0,52 and 104; mTSS was evaluated by two readers independently, in accordance with previously reported methods. $^{5} 6$ Additional analyses comparing clinical efficacy between the two groups included disease activity score (DAS) 28 (erythrocyte sedimentation rate (ESR)), simple disease activity index (SDAI), swollen joint count (SJC), tender joint count (TJC), Health Assessment Questionnaire Disability Index
(HAQ-DI), physician's and patient's global assessments of disease activity (PtGADA), patient's assessment of arthritis pain, ESR and C-reactive protein (CRP) levels. Clinical remission was defined as achieving SDAI $\leq 3.3$, DAS28(ESR) $<2.6$ or $\leq 1$ on all four of the following criteria (Boolean remission): the number of TJC (in 28 joints), number of SJC (in 28 joints), CRP (mg/dL) and PtGADA (100 $\mathrm{mm}$ visual analogue scale (VAS) data converted to $\mathrm{cm}$ ).

\section{Safety assessments}

All safety events during the PT period were recorded as adverse events (AEs) or serious AEs (SAEs). Laboratory tests (haematological, blood chemistry, urinalysis), chest radiographs and ECG were also evaluated.

\section{Statistical analyses}

Full details of the statistical analyses can be found in the online supplementary materials. In brief, the full analysis set (FAS; defined as all patients who received $\geq 1$ dose of study drug and provided any efficacy data thereafter) was used for all efficacy measurements. Missing data were imputed using linear extrapolation for mTSS and last observation carried forward (LOCF) for all other efficacy variables. Change from baseline in mTSS at weeks 52 and 104 was analysed using an analysis of covariance (ANCOVA) model. Fisher's exact test was used to compare rates of mTSS nonprogression (mTSS change from baseline $\leq 0.5$ ) and clinical remission at weeks 52 and 104, between the PBO and CZP groups.

\section{RESULTS}

\section{Patient characteristics and disposition}

Of the 316 patients who were randomised and received at least one dose of study drug (FAS population), 179 patients entered
Figure 1 Patient disposition in the Certolizumab-Optimal Prevention of joint damage for Early rheumatoid arthritis trial. CZP, certolizumab pegol; FAS, full analysis set; MTX, methotrexate; PBO, placebo.

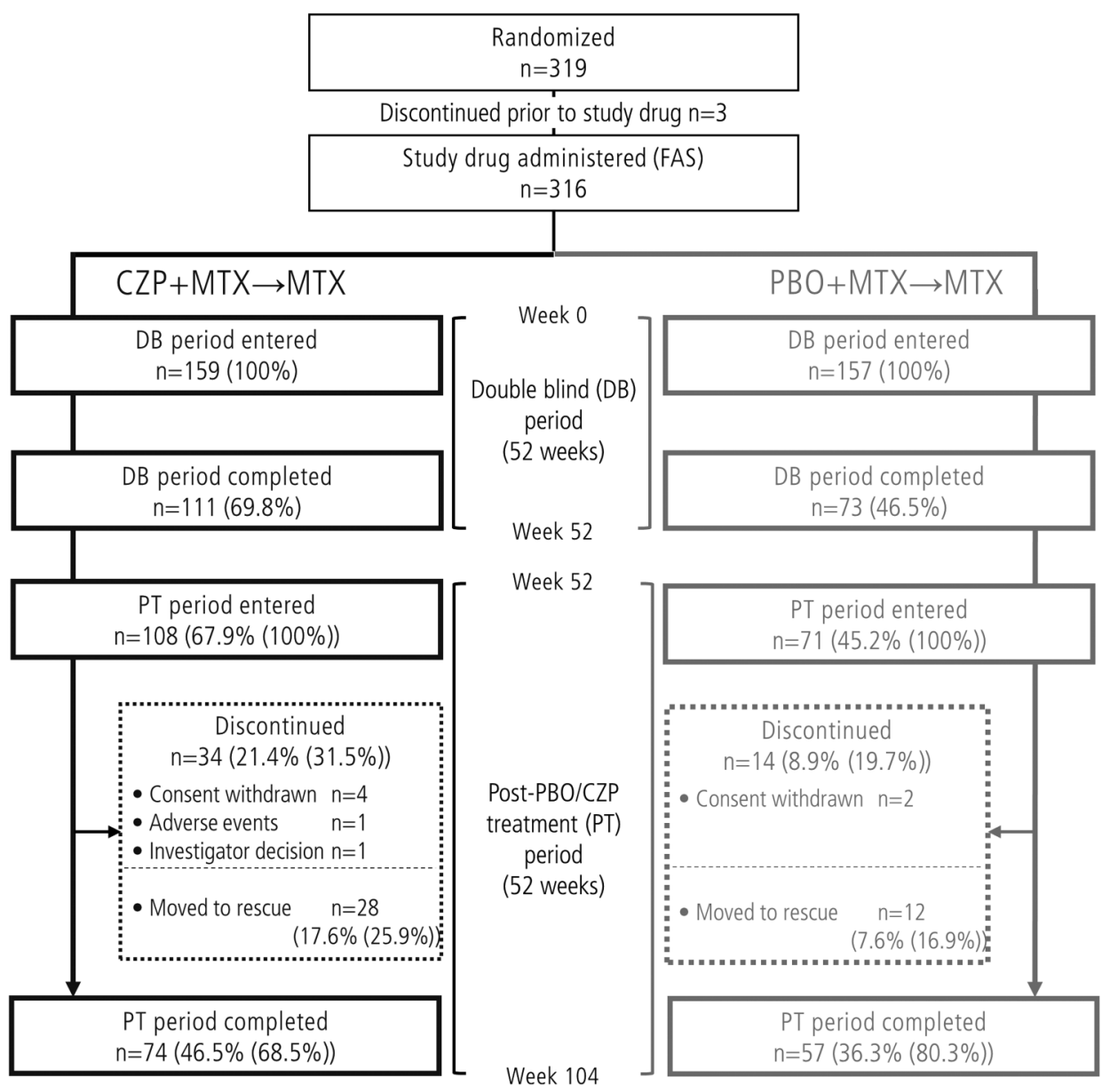

Week 104 
the PT period and 131 patients completed the study (figure 1). The proportion of patients completing the PT period (from the patients who entered the PT period) was $68.5 \%$ and $80.3 \%$ in the CZP+MTX $\rightarrow$ MTX group and the $\mathrm{PBO}+\mathrm{MTX} \rightarrow \mathrm{MTX}$ group, respectively (figure 1 ).

Patient baseline demographics and disease characteristics at DB baseline and at PT period entry (week 52) are shown in table 1 and online supplementary table S1. At DB baseline, patient demographics and disease characteristics were similar between the groups. In both groups, disease activity at baseline in the PT population was slightly lower than in the total population, and decreased from baseline through week 52 following treatment with either $\mathrm{CZP}+\mathrm{MTX}$ or $\mathrm{PBO}+\mathrm{MTX}$.

\section{Inhibition of joint damage progression in the total population}

As reported previously, at week 52 the change from baseline in mTSS was smaller in the CZP + MTX $\rightarrow$ MTX group compared with the $\mathrm{PBO}+\mathrm{MTX} \rightarrow \mathrm{MTX}$ group $(0.36 \pm 2.70$ vs $1.58 \pm 4.86$, $\mathrm{p}<0.001$ by ANCOVA), and the rate of radiographic nonprogression was higher in the $\mathrm{CZP}+\mathrm{MTX} \rightarrow \mathrm{MTX}$ group compared with the $\mathrm{PBO}+\mathrm{MTX} \rightarrow \mathrm{MTX}$ group $(82.9 \%$ vs $70.7 \%$; $\mathrm{p}<0.011$ by Fisher's exact test). ${ }^{4}$ During the PT period, through week 104, the changes from baseline (mean \pm SD) for total mTSS $(0.66 \pm 5.38$ vs $3.01 \pm 9.66(p=0.001))$, erosion score $(0.30 \pm 2.54$ vs $1.43 \pm 4.40(\mathrm{p}=0.003))$ and joint space narrowing score $(0.36 \pm 4.27$ vs $1.58 \pm 7.17(\mathrm{p}=0.002))$ were lower for the CZP + MTX $\rightarrow$ MTX group compared with the $\mathrm{PBO}+\mathrm{MTX} \rightarrow \mathrm{MTX}$ group using linear extrapolation for missing data imputation (figure $2 \mathrm{~A}$ ). A sensitivity analysis using an LOCF imputation method (figure 2A) confirmed the results of the primary analysis (linear extrapolation). At week 104, the proportion of patients with radiographic non-progression (ie, mTSS change from baseline $\leq 0.5$ ) was higher for the CZP + MTX $\rightarrow$ MTX group compared with the PBO + MTX $\rightarrow$ MTX group $(84.2 \%$ vs $67.5 \%, \mathrm{p}<0.001)$. Furthermore, the proportion of patients with rapid radiographic progression (RRP: mTSS yearly change from baseline $\geq 5$ ) at week 104 was lower for CZP+MTX $\rightarrow$ MTX group compared with the PBO + MTX $\rightarrow$ MTX group (3.2\% vs 9.6\%, p=0.022). Subgroup analyses revealed that high baseline mTSS, CRP or TNF was associated with poor week 104 radiographic outcomes in the PBO + MTX $\rightarrow$ MTX group. The CZP + MTX $\rightarrow$ MTX group also showed higher inhibition of radiographic progression in these populations (see online supplementary table S3). Consistent with radiographic findings, the proportion of patients with HAQ remission (HAQ $\leq 0.5$ ) at week 104 was numerically higher in the $\mathrm{CZP}+\mathrm{MTX} \rightarrow \mathrm{MTX}$ group than the $\mathrm{PBO}$ + MTX $\rightarrow$ MTX group $(73.0 \%$ vs $63.7 \%, p=0.09)$. In addition, the proportion of the patients who achieved HAQ remission at week 104 was higher in patients who showed non-radiographic progression at week 104 than those who did not $(76.7 \%$ vs

Table 1 Baseline demographics and patient characteristics

\begin{tabular}{|c|c|c|c|c|c|c|}
\hline & \multicolumn{3}{|c|}{$\mathrm{CZP}+\mathrm{MTX} \rightarrow \mathrm{MTX}$} & \multicolumn{3}{|c|}{$\mathrm{PBO}+\mathrm{MTX} \rightarrow \mathrm{MTX}$} \\
\hline & \multirow{2}{*}{$\begin{array}{l}\text { Total patients } \\
\mathrm{n}=159 \\
\text { DB baseline } \\
\text { (Week } 0)\end{array}$} & \multicolumn{2}{|c|}{$\begin{array}{l}\text { Patients entering PT period } \\
n=108\end{array}$} & \multirow{2}{*}{$\begin{array}{l}\text { Total patients } \\
n=157 \\
\text { DB baseline } \\
(\text { Week } 0)\end{array}$} & \multicolumn{2}{|c|}{$\begin{array}{l}\text { Patients entering PT period } \\
n=71\end{array}$} \\
\hline & & $\begin{array}{l}\text { DB baseline } \\
\text { (Week 0) }\end{array}$ & $\begin{array}{l}\text { PT baseline } \\
\text { (Week 52) }\end{array}$ & & $\begin{array}{l}\text { DB baseline } \\
\text { (Week 0) }\end{array}$ & $\begin{array}{l}\text { PT baseline } \\
\text { (Week 52) }\end{array}$ \\
\hline Age (years) & $49.4 \pm 10.6$ & $48.8 \pm 11.2$ & - & $49.0 \pm 10.3$ & $48.6 \pm 10.8$ & - \\
\hline Female, $\mathrm{n}(\%)$ & $129(81.1)$ & $85(78.7)$ & - & $127(80.9)$ & $58(81.7)$ & - \\
\hline Weight $(\mathrm{kg})$ & $57.4 \pm 11.3$ & $57.0 \pm 11.5$ & - & $57.4 \pm 10.6$ & $57.4 \pm 10.3$ & - \\
\hline BMI $\left(\mathrm{kg} / \mathrm{m}^{2}\right)$ & $22.4 \pm 3.9$ & $22.2 \pm 3.7$ & - & $22.5 \pm 3.7$ & $22.4 \pm 3.7$ & - \\
\hline RA duration (months) ${ }^{*}$ & $4.0 \pm 2.9$ & $4.4 \pm 3.1$ & - & $4.3 \pm 2.8$ & $4.4 \pm 3.1$ & - \\
\hline Anti-CCP antibody positive, $\mathrm{n}(\%)$ & $159(100.0)$ & $71(100.0)$ & - & $157(100.0)$ & $108(100.0)$ & - \\
\hline RF positive, $\mathrm{n}(\%)$ & $153(96.2)$ & $104(96.3)$ & - & $146(93.0)$ & $68(95.8)$ & - \\
\hline Bone erosion (judged by physician), $\mathrm{n}(\%)$ & $79(49.7)$ & $41(47.2)$ & - & $80(51.0)$ & $34(47.9)$ & - \\
\hline TJC (/28 joints) & $8.4 \pm 6.1$ & $7.5 \pm 5.8$ & $0.5 \pm 1.1$ & $8.9 \pm 6.5$ & $7.3 \pm 6.1$ & $0.6 \pm 1.6$ \\
\hline SJC (/28 joints) & $8.3 \pm 5.3$ & $7.6 \pm 4.6$ & $0.3 \pm 0.7$ & $8.4 \pm 5.3$ & $7.0 \pm 4.2$ & $0.4 \pm 1.4$ \\
\hline ESR (mm/hour) & $38.4 \pm 25.3$ & $36.3 \pm 23.7$ & $12.8 \pm 9.9$ & $43.7 \pm 28.2$ & $36.5 \pm 22.2$ & $15.5 \pm 14.3$ \\
\hline $\mathrm{CRP}(\mathrm{mg} / \mathrm{dL})$ & $1.29 \pm 1.82$ & $1.12 \pm 1.51$ & $0.06 \pm 0.13$ & $1.52 \pm 1.91$ & $1.03 \pm 1.39$ & $0.17 \pm 0.37$ \\
\hline MMP-3 (ng/mL) & $130.4 \pm 135.4$ & $125.3 \pm 135.4$ & $47.7 \pm 25.7$ & $185.4 \pm 214.9$ & $167.3 \pm 204.3$ & $52.5 \pm 31.1$ \\
\hline DAS28(ESR) & $5.4 \pm 1.1$ & $5.2 \pm 1.1$ & $1.9 \pm 0.8$ & $5.5 \pm 1.2$ & $5.1 \pm 1.0$ & $2.2 \pm 0.7$ \\
\hline SDAI & $28.7 \pm 12.5$ & $27.0 \pm 11.2$ & $2.4 \pm 2.6$ & $30.0 \pm 13.6$ & $24.6 \pm 11.3$ & $2.7 \pm 3.1$ \\
\hline HAQ-DI score & $1.01 \pm 0.64$ & $1.04 \pm 0.63$ & $0.14 \pm 0.26$ & $1.05 \pm 0.69$ & $0.79 \pm 0.57$ & $0.07 \pm 0.14$ \\
\hline mTSS & $4.1 \pm 7.4$ & $3.8 \pm 7.4$ & $3.7 \pm 7.4$ & $5.5 \pm 15.0$ & $3.2 \pm 6.2$ & $3.4 \pm 6.3$ \\
\hline Erosion score & $1.9 \pm 4.0$ & $1.6 \pm 3.9$ & $1.6 \pm 3.7$ & $2.5 \pm 7.8$ & $1.6 \pm 3.3$ & $1.8 \pm 3.2$ \\
\hline Joint space narrowing score & $2.1 \pm 4.6$ & $2.2 \pm 4.8$ & $2.2 \pm 4.8$ & $2.9 \pm 8.3$ & $1.5 \pm 4.0$ & $1.6 \pm 4.1$ \\
\hline Average weekly MTX dose (mg/week)† & $11.4 \pm 3.1$ & $11.3 \pm 3.2$ & $10.9 \pm 4.1$ & $11.5 \pm 2.8$ & $11.5 \pm 3.1$ & $11.1 \pm 3.7$ \\
\hline
\end{tabular}

Values are mean \pm SD unless otherwise indicated. Data in DB baseline columns represent average during weeks 0-104, whereas data in PT baseline columns represent average during weeks 52-104.

*Time from onset of persistent arthritic symptoms.

†MTX dose was initiated at $8 \mathrm{mg} /$ week and escalated to the maximum tolerated dose (up to $16 \mathrm{mg} / \mathrm{week}$ ) by week 8 .

BMI, body mass index; CCP, cyclic citrullinated peptide; CRP, C reactive protein; CZP, certolizumab pegol; DB, double blind; ESR, erythrocyte sedimentation rate; HAQ-DI, Health

Assessment Questionnaire Disability Index; MMP-3, matrix metalloproteinase-3; mTSS, modified total Sharp score; MTX, methotrexate; PBO, placebo; PT, post treatment; RA, rheumatoid arthritis; RF, rheumatoid factor; SJC, swollen joint count; TJC, tender joint count. 


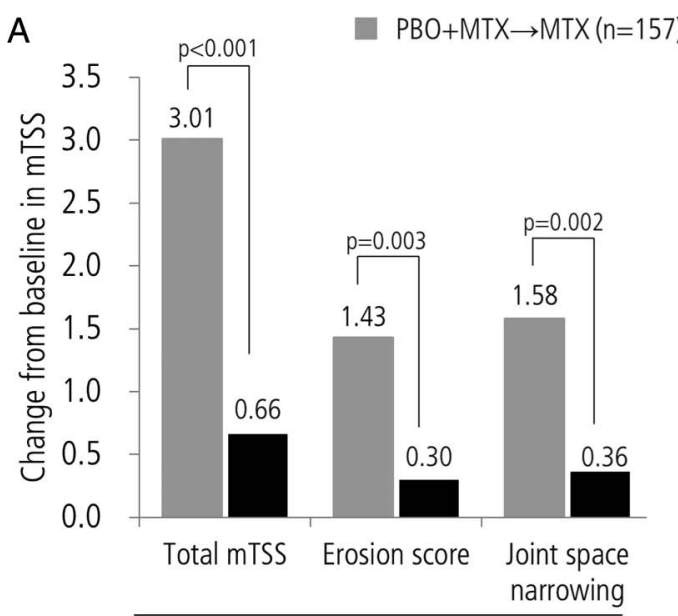

Linear Extrapolation

$C Z P+M T X \rightarrow M T X(n=158)$

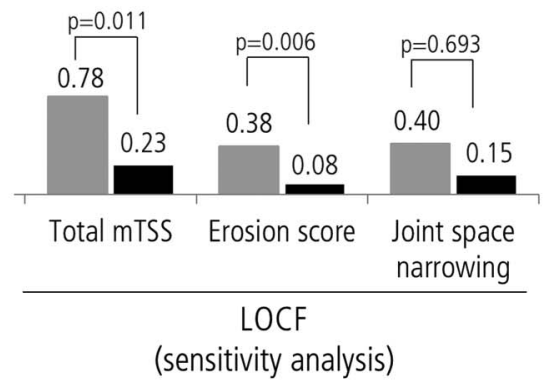

B

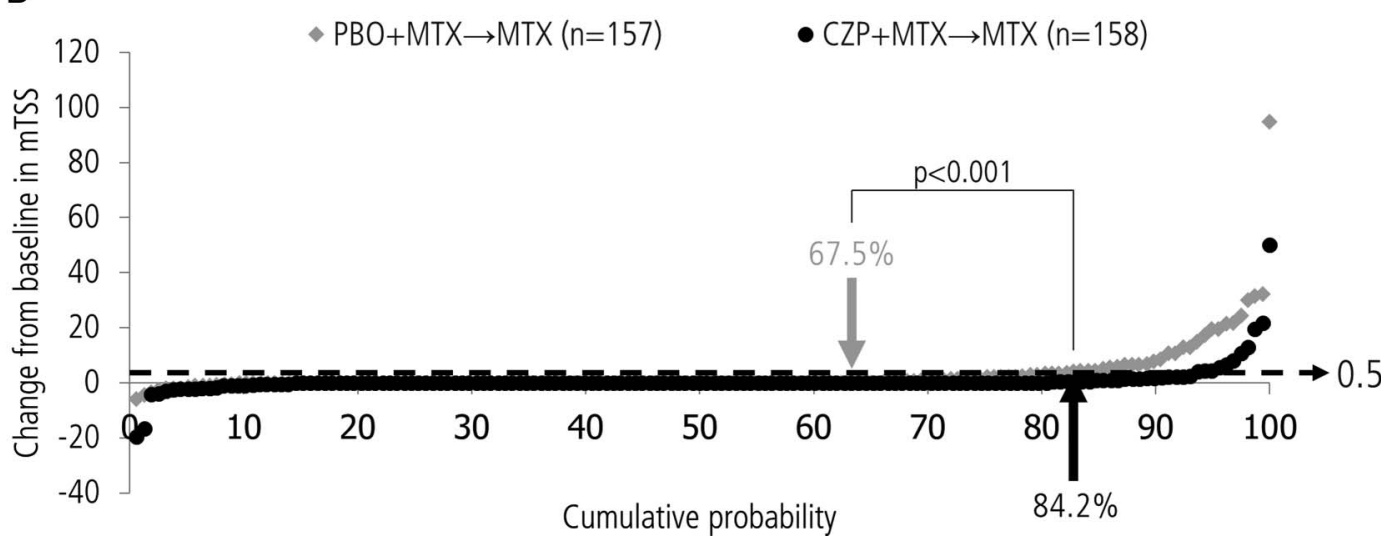

Figure 2 (A) Change from baseline in modified total Sharp score (mTSS) at week 104. (B) Cumulative probability plot of mTSS change from baseline at week 104 for both PBO+MTX $\rightarrow$ MTX and CZP+MTX $\rightarrow$ MTX groups. Change from baseline in mTSS was analysed using an analysis of covariance model in which actual scores were converted to rank scores, using the treatment group as a factor and baseline rank score as a covariate. Rate of mTSS non-progression (mTSS change from baseline $\leq 0.5$ ) was compared using Fisher's exact test. CZP, certolizumab pegol; LOCF, last observation carried forward; MTX, methotrexate; PBO, placebo.

$52.0 \%(\mathrm{p}=0.015)$ in $\mathrm{CZP}+\mathrm{MTX} \rightarrow \mathrm{MTX}$, and $70.8 \%$ vs $49.0 \%$ $(\mathrm{p}=0.013)$ in $\mathrm{PBO}+\mathrm{MTX} \rightarrow \mathrm{MTX})$.

\section{Clinical remission in the total population}

The numbers of patients achieving SDAI, Boolean and DAS28 (ESR) remission were calculated throughout both the DB and PT periods (figure 3). At the end of the DB period (week 52), clinical remission rates were significantly higher in the CZP $+\mathrm{MTX} \rightarrow \mathrm{MTX}$ group compared with the $\mathrm{PBO}+\mathrm{MTX} \rightarrow \mathrm{MTX}$ group. ${ }^{4}$

The rates of clinical remission observed for the CZP + MTX $\rightarrow$ MTX group decreased during the first 16 weeks of the PT period; however, the rate stabilised from week 68 (week 16 of the PT period) through week 104. The remission rates of the $\mathrm{PBO}+\mathrm{MTX} \rightarrow \mathrm{MTX}$ group during the PT period were similar to the DB period, with no change in clinical remission observed during the transition from the end of DB to the PT period. Throughout the duration of the PT period, the rates of clinical remission remained higher in the $\mathrm{CZP}+\mathrm{MTX} \rightarrow \mathrm{MTX}$ group compared with the $\mathrm{PBO}+\mathrm{MTX} \rightarrow \mathrm{MTX}$ group $(41.5 \%$ vs $29.3 \%$ $(p=0.026), 34.6 \%$ vs $24.2 \%(p=0.049)$ and $41.5 \%$ vs $33.1 \%$ $(\mathrm{p}=0.132)$ at week 104 in SDAI, Boolean and DAS28(ESR), respectively). The proportion of patients with low disease activity (DAS28(ESR) $\leq 3.2$ ) was also higher in the CZP + MTX $\rightarrow$ MTX group compared with the $\mathrm{PBO}+\mathrm{MTX} \rightarrow \mathrm{MTX}$ group throughout the PT period (see online supplementary figure S1).

\section{Impact of CZP discontinuation in the CZP+MTX $\rightarrow$ MTX group}

The impact of CZP discontinuation was assessed on patients who entered the PT period (PT population) from the CZP + MTX group $(n=108)$. Of these, 74 patients $(68.5 \%)$ completed the 1-year PT period with MTX therapy (figure 4A). Rates of radiographic non-progression during the PT period (94.4\% (102/108); figure 4B) were similar to the rates observed during the DB period $(91.7 \%(99 / 108))$, as were mean changes in mTSS $( \pm S D)$ during the first and second 52-week periods (DB period (weeks 0-52): $-0.03( \pm 0.80)$; PT period (weeks 52 $-104)$ : $0.06( \pm 0.76))$. Conversely, clinical remission rates in the PT population of the CZP + MTX $\rightarrow$ MTX group showed decreases in SDAI, Boolean and DAS28(ESR) definitions of 
A

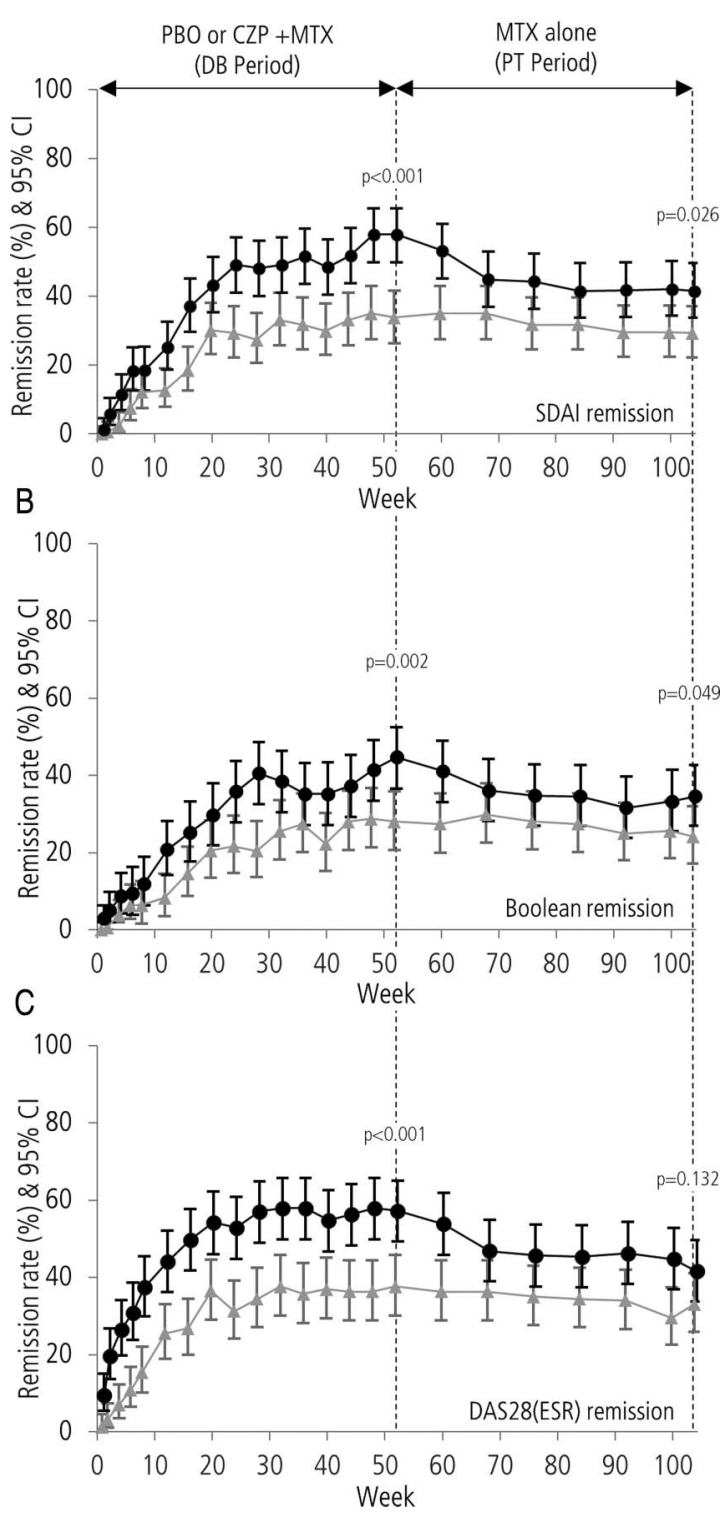

Figure 3 The proportion of patients achieving (A) SDAI remission, (B) Boolean remission and (C) DAS28(ESR) remission during the Certolizumab-Optimal Prevention of joint damage for Early rheumatoid arthritis study in both the PBO+MTX $\rightarrow$ MTX and CZP+MTX $\rightarrow$ MTX groups. Remission rates at weeks 52 and 104 were compared using Fisher's exact test. CZP, certolizumab pegol; DB, double blind; ESR, erythrocyte sedimentation rate; MTX, methotrexate; PBO, placebo; PT, post treatment.

remission from weeks 52 to 104 (from $79.6 \%$ to $55.6 \%, 61.1 \%$ to $46.3 \%, 77.8 \%$ to $54.6 \%$, respectively; figure $4 \mathrm{C}$ ).

\section{Retreatment of CZP after symptom flare in CZP+MTX $\rightarrow$ MTX group}

Of the 34 patients who withdrew during the PT period, 28 patients moved to rescue treatment, restarting CZP therapy as a consequence of symptom flares (figure 1). Improvements were observed in this cohort of patients (figure 4D); mean disease activity decreased from DAS28(ESR) 4.40 at CZP restart $(n=28)$ to 2.49 after 12 weeks $(n=25$; observed case). Of the 28 patients receiving rescue treatment, 26 continued retreatment until the end of the study. The majority of patients receiving
CZP retreatment for $\geq 12$ weeks $(n=25)$ responded positively; using DAS28(ESR) criteria, 24/25 achieved low disease activity and $21 / 25$ achieved remission on at least one study visit.

\section{Safety}

Study drug exposure during both the total study period and the PT period was higher for the CZP + MTX $\rightarrow$ MTX group (total: 223.6 patient-years (PY), PT: 87.7 PY) compared with the PBO + MTX $\rightarrow$ MTX group (total: 179.4 PY, PT: 63.4 PY; table 2). This difference could be attributed to the higher withdrawal rate in the $\mathrm{PBO}+\mathrm{MTX} \rightarrow \mathrm{MTX}$ group. Overall, no clinically relevant difference was observed in the total incidence of AEs between the CZP + MTX $\rightarrow$ MTX group and the $\mathrm{PBO}$ + MTX $\rightarrow$ MTX group through week 104 (154 patients (96.9\%) vs 150 patients $(95.5 \%))$, or SAEs (17 patients (10.7\%) vs 18 patients $(11.5 \%)$ ). Incidence rates of some types of AEs including infections and infestations, pneumonia and hepatic disorders were higher during the DB period (weeks 0-52) compared with the PT period (weeks 52-104); however, this increase was observed for both the CZP+MTX $\rightarrow$ MTX and the $\mathrm{PBO}$ + MTX $\rightarrow$ MTX group (table 2 and see online supplementary table S2).

\section{DISCUSSION}

Biological DMARDs are considered second-line therapies for patients who cannot achieve treatment targets using conventional synthetic disease-modifying antirheumatic drugs (csDMARDs) in the management of RA. ${ }^{7}$ However, it has been reported that the inhibitory effect of bDMARDs on joint damage is superior to that of csDMARDs, including MTX. ${ }^{4}$ Although there is some evidence of bone erosion repair following treatment with bDMARDs, ${ }^{9}$ joint destruction in patients with RA is generally considered to be irreversible. ${ }^{10}$ Consequently, prevention of significant joint damage is crucial to avoid permanent functional disability, ${ }^{11}$ supporting early treatment with bDMARDs.

Concerns have been raised that initiating aggressive treatment with a bDMARD may be excessive for some patients and so identifying patients who would particularly benefit from initial aggressive treatment is critical when considering it. The feasibility of bDMARD withdrawal after achieving a therapeutic target is also of importance from both safety and economical points of view. If these issues are overcome, there is the possibility of a clinical approach where RA therapy is initiated with a bDMARD in the early stage of disease, leading to improved outcomes that can be maintained even after withdrawal of the initial aggressive treatment. ${ }^{12}$

C-OPERA was designed to assess the clinical benefit of CZP treatment concomitant with MTX as first-line therapy for early RA, particularly for patients who were considered to require aggressive treatment. Patients who had poor prognostic factors, including a high titre of anticyclic citrullinated peptide antibody in addition to either rheumatoid factor positivity or bone erosions, were eligible to enter the study. C-OPERA was a study composed of two periods. The results from the first year of the study demonstrated the clinical benefit of adding CZP to MTX therapy (DAS28(ESR) remission and radiographic nonprogression was achieved in more than $50 \%$ and $80 \%$ of patients, respectively), suggesting that the introduction of CZP at a very early stage led to substantial therapeutic effects, despite poor prognosis. ${ }^{4}$ In this report, we assessed whether the clinical benefit of initial 1-year CZP +MTX treatment was observed after stopping CZP and continuing with MTX therapy for 1 year. 
Figure 4 (A) Retention rate after discontinuation of CZP (Kaplan-Meier plot), (B) modified total Sharp score non-progression rate during and after 52-week CZP therapy for patients who entered the PT period from the CZP +MTX group, (C) clinical remission at weeks 52 and 104, and (D) DAS28 (ESR) over time during DB period and after CZP restart in patients who were retreated with CZP $(n=28)$. CZP, certolizumab pegol; DB, double blind; ESR, erythrocyte sedimentation rate; MTX, methotrexate; PT, post treatment.
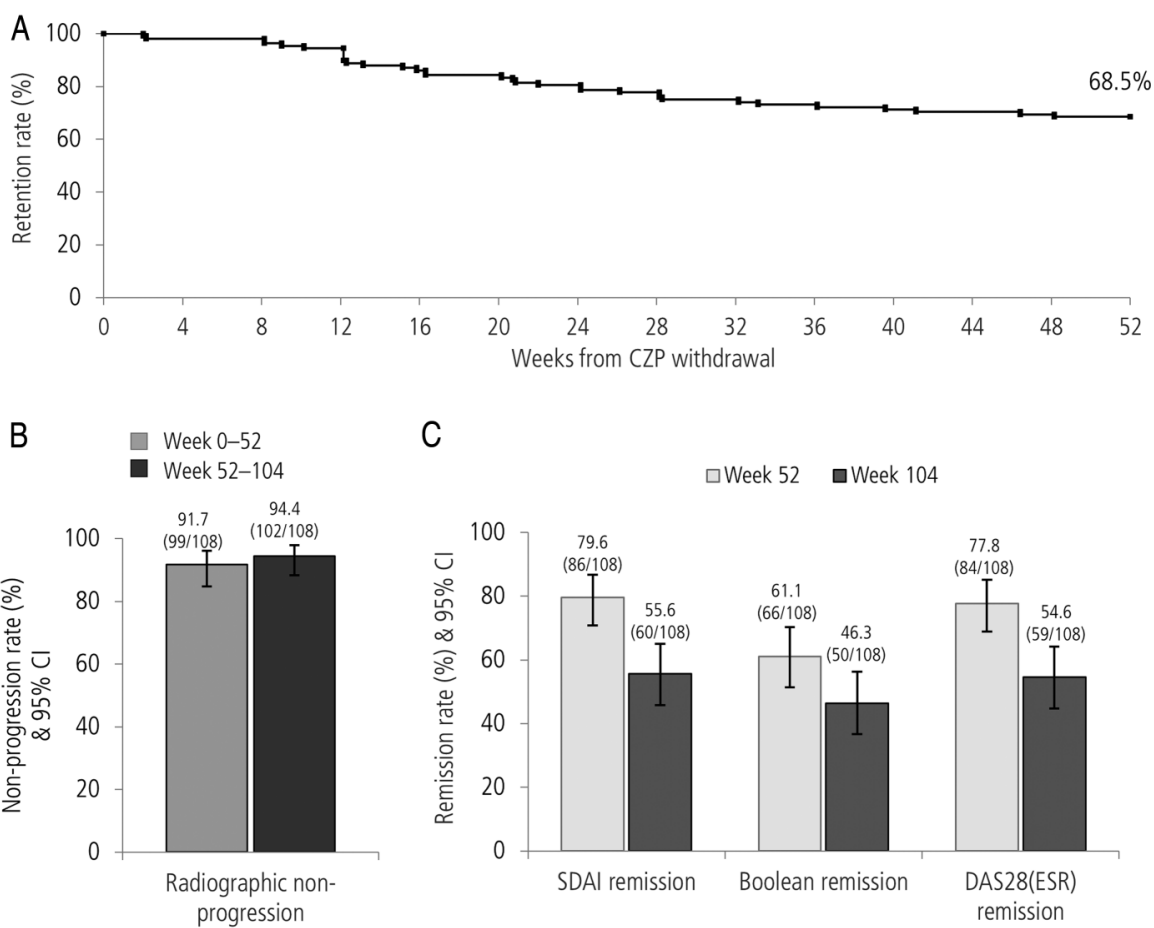

C
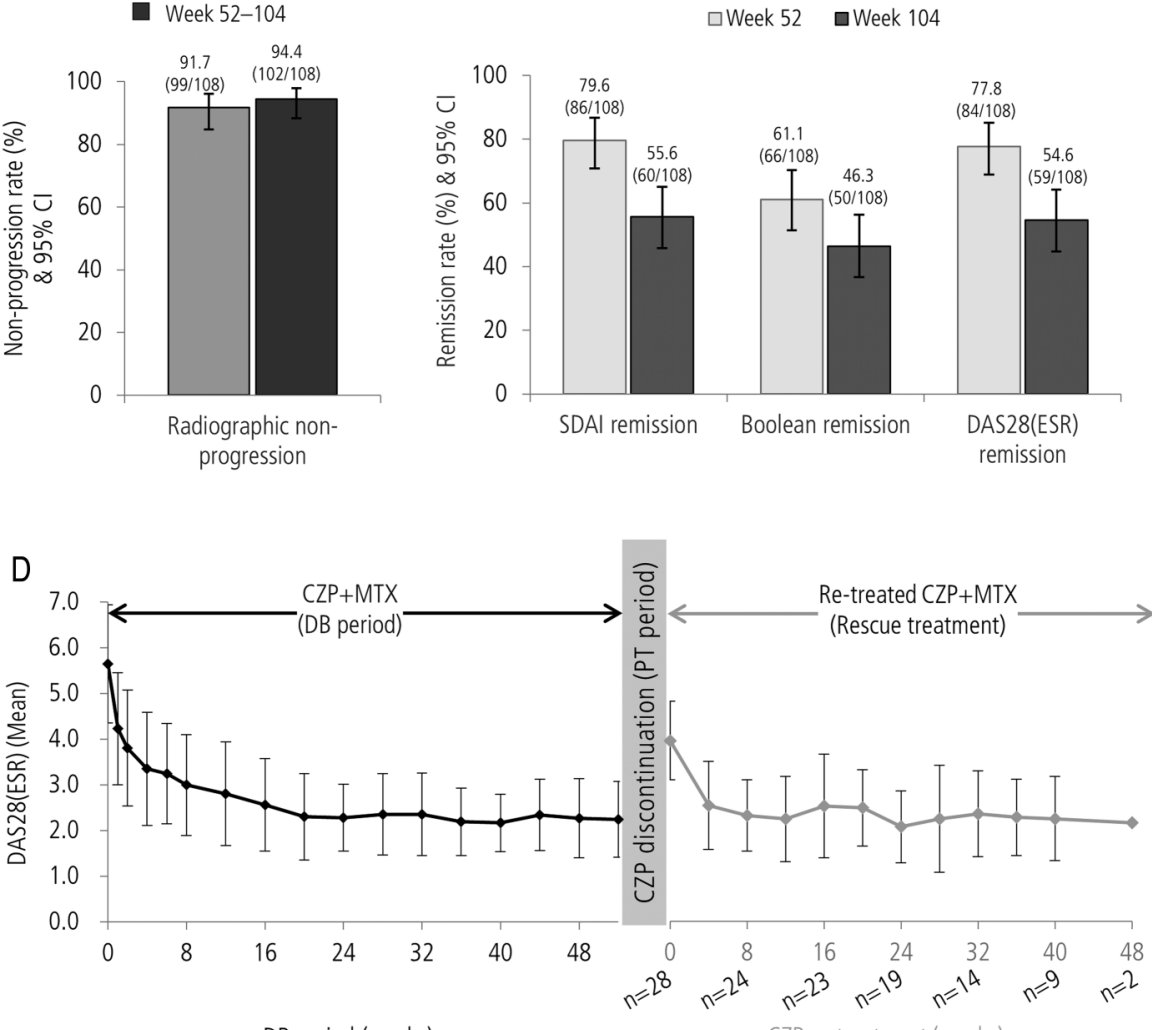

DB period (weeks)
A key finding from the PT period reported here, was that the beneficial effect seen after an initial 1 year of treatment with CZP was observed after discontinuing CZP therapy when the dose of MTX remained optimised. Radiographic progression, estimated using linear extrapolation, remained lower in the CZP + MTX $\rightarrow$ MTX group compared with the $\mathrm{PBO}+\mathrm{MTX} \rightarrow \mathrm{MTX}$ group. When using linear extrapolation, patient withdrawal can result in an overestimation of mTSS change from baseline. To overcome this, analyses were repeated using LOCF imputation, confirming the efficacy of CZP co-administration. The rate of radiographic non-progression observed in the CZP + MTX $\rightarrow$ MTX patient population during the PT period was similar to that observed during the DB period in the same population. This suggests that joint destruction may be prevented even after discontinuation of CZP in patients who responded adequately to initial treatment with $\mathrm{CZP}+\mathrm{MTX}$. These data, combined with the lower rate of RRP in the CZP $+\mathrm{MTX} \rightarrow \mathrm{MTX}$ group compared with the PBO $+\mathrm{MTX} \rightarrow \mathrm{MTX}$, and the subgroup analyses (see online supplementary table S3) suggest that induction treatment with $\mathrm{CZP}+\mathrm{MTX}$ benefits those with a high risk of radiographic progression. The rate of HAQ remission was higher in patients with radiographic non- progression compared with those with radiographic progression, suggesting that radiographic progression associates with functional disability even during the 2 years of the study. These results suggest that early treatment with CZP during the initial stages of the disease, when rapid joint damage may take place, ${ }^{13}$ could prevent long-term progression of joint damage and functional disability as previously suggested in the 'window of opportunity' concept. $^{8}$ 14-16

In addition to joint damage prevention, the rates of clinical remission throughout the PT period remained higher in the CZP $+\mathrm{MTX} \rightarrow \mathrm{MTX}$ group compared with the $\mathrm{PBO}+\mathrm{MTX} \rightarrow \mathrm{MTX}$ group. After CZP discontinuation, approximately 25\% of the patients flared; however, they showed rapid response to CZP retreatment, with recovery to preflare disease activity levels. Although joint destruction was consistently prevented in the PT population following CZP withdrawal, clinical remission was sometimes lost. Discrepancies in clinical and radiographic efficacy have been reported for adalimumab (ADA); ${ }^{17}$ similar differences in the clinical and radiographic efficacies of CZP that continue following treatment discontinuation could be responsible for the results observed here. A decrease in remission rate was mainly observed during the first 16 weeks after CZP 
Table 2 Summary of treatment-emergent adverse events (TEAE)

\begin{tabular}{|c|c|c|c|c|c|c|}
\hline & \multicolumn{3}{|c|}{$\mathrm{CZP}+\mathrm{MTX} \rightarrow \mathrm{MTX}$} & \multicolumn{3}{|c|}{$\mathrm{PBO}+\mathrm{MTX} \rightarrow \mathrm{MTX}$} \\
\hline & $\begin{array}{l}\text { Week 0-52 } \\
\text { CZP+MTX } \\
n=159\end{array}$ & $\begin{array}{l}\text { Week 52-104 } \\
\text { MTX } \\
n=108\end{array}$ & $\begin{array}{l}\text { Week } 0-104 \\
\text { CZP+MTX } \rightarrow \text { MTX } \\
n=159\end{array}$ & $\begin{array}{l}\text { Week 0-52 } \\
\text { PBO+MTX } \\
n=157\end{array}$ & $\begin{array}{l}\text { Week 52-104 } \\
\text { MTX } \\
n=71\end{array}$ & $\begin{array}{l}\text { Week 0-104 } \\
\text { PBO+MTX } \rightarrow \text { MTX } \\
n=157\end{array}$ \\
\hline AE summary ${ }^{\dagger}$ & $\mathrm{n}(\%)$ & $\mathrm{n}(\%)$ & $\mathrm{n}(\%)$ & $\mathrm{n}(\%)$ & $\mathrm{n}(\%)$ & $\mathrm{n}(\%)$ \\
\hline Any AEs & $153(96.2)$ & $85(78.7)$ & $154(96.9)$ & $148(94.3)$ & $57(80.3)$ & $150(95.5)$ \\
\hline Event rate* & 11.0 & 6.8 & 9.4 & 12.9 & 6.3 & 10.6 \\
\hline Deaths & 0 & 0 & 0 & 0 & 0 & 0 \\
\hline AEs of interest $t$ & n (\%) & n (\%) & n (\%) & n (\%) & n (\%) & n (\%) \\
\hline Infections and infestations & $97(61.0)$ & $45(41.7)$ & $114(71.7)$ & $87(55.4)$ & $30(42.3)$ & $93(59.2)$ \\
\hline Malignancies & $1(0.6) \ddagger$ & $1(0.9) \ddagger$ & $2(1.3) \ddagger$ & 0 & 0 & 0 \\
\hline Hepatic disorders§ & $68(42.8)$ & $12(11.1)$ & 73 (45.9) & 69 (43.9) & $9(12.7)$ & 73 (46.5) \\
\hline
\end{tabular}

*Number of events per 100 patient-years.

$\mathrm{tn}=$ number of subjects reporting at least one TEAE within System Organ Class/Preferred Term.

$\ddagger$ Cervix carcinoma.

§Including following preferred terms: alanine aminotransferase increased, aspartate aminotransferase increased, $\gamma$-glutamyltransferase increased, hepatic function abnormal, hepatic enzyme increased, hepatic steatosis, hyperbilirubinaemia, liver disorder, liver function test abnormal; MedDRA V.14.1.

$A E$, adverse events; CZP, certolizumab pegol; MTX, methotrexate; PBO, placebo.

discontinuation. We speculate that patients who still produce excessive TNF may have flared as the concentration of CZP decreased.

Overall, the results suggest that initial aggressive treatment with a bDMARD could be a potential treatment option at the early stage of disease, especially for patients who have a poor prognosis. Once patients achieve their treatment targets, bDMARD treatment could be withdrawn. This treatment approach has the potential to prevent irreversible joint damage, reduce patient risk of AEs and be a more cost-effective way to manage RA over the long term. This approach is supported by two further studies. The Optimal Protocol for Methotrexate and Adalimumab Combination Therapy in Early Rheumatoid Arthritis (OPTIMA) study demonstrated minimal loss of clinical response without significant radiographic progression after ADA withdrawal in patients with early RA who initiated combination therapy with MTX. ${ }^{8}$ While, The High Induction Therapy with Anti-Rheumatic Drugs (HIT HARD) study showed better radiographic outcomes but no significant difference in disease activity after ADA withdrawal in the ADA + MTX $\rightarrow$ MTX group compared with MTX alone. ${ }^{18}$ Differences in the results of these studies compared with C-OPERA suggest that the condition of the 'induction-maintenance' regimen ${ }^{12}$ may be important. Further studies are now needed to identify the appropriate disease activity targets required to achieve continued disease inhibition following CZP withdrawal, and to identify patient populations that would particularly benefit from first-line therapy with CZP. Moreover, additional analyses are also required to confirm whether this approach has significant clinical benefits for patients failing to respond to MTX therapy after 3 months, which is the approach currently recommended in treat-to-target guidelines. ${ }^{19}$

Safety analyses showed similar rates of SAEs for both the CZP $+\mathrm{MTX} \rightarrow \mathrm{MTX}$ and $\mathrm{PBO}+\mathrm{MTX} \rightarrow \mathrm{MTX}$ groups over the 2 years of the C-OPERA study, indicating that there are no major safety concerns when adding CZP to optimised MTX therapy. Incidences of AEs and SAEs during the PT period were lower compared with the initial DB period in both groups. One reason for this may be 'survival bias', where patients discontinued the study because of an intolerance to the study drugs (CZP and/or MTX) in the first period, resulting in a lower $\mathrm{AE}$ rate in the second. ${ }^{20}$

This study has several limitations. In clinical practice, only patients failing to respond to MTX would receive CZP therapy, and so it is not known how this approach compares with initial CZP therapy. No patients received CZP for a full 2 years or were treated with a reduced dose of CZP, so it was not possible to compare these treatment regimens with CZP discontinuation. CZP withdrawal had not been optimised; therefore, there is the potential for further investigation regarding the appropriate treatment targets and the timing of CZP withdrawal in different patient populations. There were differences between the study design of C-OPERA and current RA treatment recommendations. For example, in clinical practice, treatment recommendations for patients with poor prognostic factors include using additional DMARDs in addition to MTX, ${ }^{7}{ }^{21}$ which was prohibited in C-OPERA. C-OPERA was not designed as an intercontinental global study; thus, it is not known whether these results are generalisable to ethnicities other than Japanese. In particular, the MTX dose of $16 \mathrm{mg}$ is low compared with similar RA studies from the European Union and USA (15-17 mg/week). ${ }^{22-26}$ However, when considering differences in patient body weight and MTX metabolism, ${ }^{27}$ a lower dose of MTX in this study may correspond to the doses used in those previous studies. Finally, as non-responding patients were eligible to receive rescue treatment from 24 weeks onwards, we cannot exclude the possibility that a proportion of the $70 \mathrm{PBO}+\mathrm{MTX}$ patients switching to rescue therapy in the first year may have achieved clinical response if treated for a longer period of time. ${ }^{4}$ 
Overall, these results suggest that patients with early RA would benefit from the addition of CZP to MTX therapy during the early stages of disease, particularly with respect to the prevention of joint destruction. Although this aggressive therapeutic strategy would not be recommended for all patients, it may be a potential option for those patients with a high risk of experiencing rapid joint destruction. How to identify these patients at disease onset requires further investigation.

\section{Author affiliations}

${ }^{1}$ Hokkaido University Graduate School of Medicine, Sapporo, Japan

${ }^{2}$ The First Department of Internal Medicine, University of Occupational and Environmental Health, Kitakyushu, Fukuoka, Japan

${ }^{3}$ Department of Allergy and Rheumatology, Graduate School of Medicine, The University of Tokyo, Tokyo, Japan

${ }^{4}$ Division of Rheumatology, Department of Internal Medicine, Keio University School of Medicine, Tokyo, Japan

${ }^{5}$ Tokyo Women's Medical University, Tokyo, Japan

${ }^{6}$ Nagoya University Graduate School and Faculty of Medicine, Nagoya, Japan

${ }^{7}$ Rheumatic and Collagen Disease Center, Sasebo Chuo Hospital, Sasebo, Nagasaki, Japan

${ }^{8}$ Tohoku University, Sendai, Japan

${ }^{9}$ Division of Biostatistics and Clinical Epidemiology, The University of Toyama

Graduate School of Medicine, Toyama, Japan

${ }^{10}$ Hiroshima Rheumatology Clinic, Hiroshima, Japan

${ }^{11}$ Yokohama Rosai Hospital, Yokohama, Japan

${ }^{12}$ Matsubara Mayflower Hospital, Kato, Japan

${ }^{13}$ Division of Rheumatology and Clinical Immunology, Jichi Medical University,

Tochigi, Japan

${ }^{14}$ UCB Pharma, Tokyo, Japan

${ }^{15}$ Astellas Pharma Inc., Tokyo, Japan

${ }^{16}$ Department of Rheumatology, Leiden University Medical Centre, Leiden, The Netherlands

${ }^{17}$ Department of Rheumatology, Tokyo Medical and Dental University, Tokyo, Japan

${ }^{18}$ Sapporo Medical Center NTT EC, Sapporo, Hokkaido, Japan

Correction notice This article has been corrected since it published Online First. Figure 2 has been updated as well as the legend for figure 4 .

Acknowledgements The authors acknowledge Lilia Marinova MD, PhD, UCB Pharma, UK, for publication coordination, and Simon Foulcer, $\mathrm{PhD}$, and Danielle Machin, BSc, from Costello Medical Consulting, Cambridge, UK, for medical writing and editorial assistance in preparing this manuscript for publication, based on the authors' input and direction. UCB Pharma reviewed only for scientific and legal accuracy.

Contributors All authors were involved in the C-OPERA study, reviewed and interpreted the data, developed the manuscript and approved the final draft.

Funding Astellas Pharma. and UCB Pharma funded this study and manuscript.

Competing interests TA has taken part in speakers' bureaus for Astellas, Bristol-Myers, Chugai and Mitsubishi-Tanabe. KY has received consultancy fees from Abbott, BMS, Chugai, Eisai, Mitsubishi-Tanabe, Pfizer, Roche and UCB Pharma; and has received research grants from Abbott, Eisai, Mitsubishi-Tanabe, Pfizer, Santen and UCB Pharma. TT has received consultancy fees from AstraZeneca, Asahi Kasei, Eli Lilly, Mitsubishi-Tanabe and Novartis; research grants from Abbott, Astellas, BMS, Chugai, Daiichi-Sankyo, Eisai, Janssen, Mitsubishi-Tanabe, Nippon Shinyaku, Otsuka, Pfizer, Sanofi-Aventis, Santen, Takeda and Teijin; and has taken part in speakers' bureaus for Abbott, BMS, Chugai, Eisai, Janssen, Mitsubishi-Tanabe, Pfizer and Takeda and UCB Pharma. HY has received consultancy fees from Abbott, Astellas, BMS, Chugai, Eisai, Janssen, Mitsubishi-Tanabe, Pfizer, Takeda and UCB Pharma; and has received research grants from Abbott, Astellas, BMS, Chugai, Eisai, Janssen, Mitsubishi-Tanabe, Pfizer, Takeda and UCB Pharma. NI has received research grants from Abbott, Astellas, BMS, Takeda, Chugai, Eisai, Janssen, Kaken Mitsubishi-Tanabe and Pfizer; and has taken part in speakers' bureaus for Abbott, Astellas, BMS, Chugai, Eisai, Janssen, Kaken, Mitsubishi-Tanabe, Otsuka, Pfizer, Taisho-Toyama and Takeda. YT has received research grants from Astellas, Abbvie, BMS, Chugai, Daiichi-Sankyo, Mitsubishi-Tanabe, MSD; has received consultancy fees from Abbott, Abbvie, Asahi Kasei, Astellas, AstraZeneca, Chugai, Daiichi-Sankyo, Eisai, Eli Lilly, GSK, Janssen, Mitsubishi-Tanabe, MSD, Pfizer, Quintiles, Takeda and UCB Pharma; and has taken part in speakers' bureaus for Abbott, Abbvie, Asahi Kasei, Astellas, AstraZeneca, Chugai, Daiichi-Sankyo, Eisai, Eli Lilly, GSK, Janssen, Mitsubishi-Tanabe, MSD, Pfizer, Quintiles, Takeda and UCB Pharma. KE has received consultancy fees from UCB Pharma. AW has received research grants from Daiichi-Sankyo, Dainippon-Sumitomo, Kyorin, Meiji Seika; Shionogi, Taiho, Taisho and Toyama Chemical; and has taken part in speakers' bureaus for Daiichi-Sankyo, Dainippon-Sumitomo, GSK, Mitsubishi-Tanabe, MSD, Pfizer, Shionogi and Taisho-Toyama. HO has received consultancy fees from Astellas and UCB Pharma. SY has received research grant from BMS and taken part in speakers' bureaus for Abbvie, Astellas, Chugai, Eizai, Pfizer, Mitsubishi-Tanabe and Takeda. YY has no competing interests to disclose. YK has received speakers' bureau from Astellas, Chugai and Ono. TM has received speaker honoraria from Pfizer Japan, Janssen Pharmaceutical Co.. and Astellas Pharma; and research grants form Quintiles Transnational Japan K.K, Janssen Pharmaceutical Co., Takeda Chemical Industries, Daiichi Sankyo Co., Astellas Pharma, Eli Lilly Japan K.K., MSD Co., Nippon Kayaku Co., Parexel International, Pfizer Japan and Bristol-Myers Squibb. MI has received payment for lectures from Astellas, Chugai, Ono and

Tanabe-Mitsubishi; has received research grants from Pfizer and a royalty fee from Chugai. TS is an employee of UCB Pharma; TO is an employee of Astellas. DvdH has received consultancy fees from Abbvie, Amgen, AstraZeneca, Augurex, BMS, Boehringer Ingelheim, Celgene, Centocor, Chugai, Covagen, Daiichi-Sankyo, Eli-Lilly, Galapagos, GSK, Janssen Biologics, Merck, Novartis, Novo-Nordisk, Otsuka, Pfizer, Roche, Sanofi-Aventis, Schering-Plough, UCB Pharma and Vertex; and is the Director of Imaging Rheumatology bv. NM has received research grants from Abbott, Astellas, Chugai, Eisai, Mitsubishi-Tanabe, Pfizer and Takeda. TK has received consultancy fees from Abbvie, Astellas, BMS, Chugai, Daiichi-Sankyo, Eisai, Mitsubishi-Tanabe, Pfizer, Santen, Taisho-Toyama, Takeda, Teijin and UCB Pharma, and has taken part in speakers' bureaus for Abbott, Astellas, BMS, Chugai, Daiichi-Sankyo, Eisai, Mitsubishi-Tanabe, Pfizer, Santen, Taisho-Toyama, Takeda, Teijin and UCB Pharma.

Ethics approval This study was conducted after review and approval by the institutional review board designated by each study site after consideration of the ethical, scientific and medical justification for the conduct of the study.

Provenance and peer review Not commissioned; externally peer reviewed.

Open Access This is an Open Access article distributed in accordance with the Creative Commons Attribution Non Commercial (CC BY-NC 4.0) license, which permits others to distribute, remix, adapt, build upon this work non-commercially, and license their derivative works on different terms, provided the original work is properly cited and the use is non-commercial. See: http://creativecommons.org/ licenses/by-nc/4.0/

\section{REFERENCES}

1 McInnes IB, Schett G. The pathogenesis of rheumatoid arthritis. N Engl J Med 2011;365:2205-19.

2 Finckh $\mathrm{A}$, Liang $\mathrm{MH}$, van Herckenrode $\mathrm{CM}$, et al. Long-term impact of early treatment on radiographic progression in rheumatoid arthritis: A meta-analysis. Arthritis Rheum 2006:55:864-72.

3 Lard LR, Visser H, Speyer I, et al. Early versus delayed treatment in patients with recent-onset rheumatoid arthritis: comparison of two cohorts who received different treatment strategies. Am J Med 2001;111:446-51.

4 Atsumi T, Yamamoto K, Takeuchi T, et al. The first double-blind, randomised, parallel-group certolizumab pegol study in methotrexate-naive early rheumatoid arthritis patients with poor prognostic factors, C-OPERA, shows inhibition of radiographic progression. Ann Rheum Dis 2016;75:75-83.

5 van der Heijde D. How to read radiographs according to the Sharp/van der Heijde method. J Rheumatol 2000:27:261-3.

6 van der Heijde DM, van Riel PL, Nuver-Zwart IH, et al. Effects of hydroxychloroquine and sulphasalazine on progression of joint damage in rheumatoid arthritis. Lancet 1989;1:1036-8.

7 Smolen JS, Landewé $\mathrm{R}$, Breedveld FC, et al. EULAR recommendations for the management of rheumatoid arthritis with synthetic and biological disease-modifying antirheumatic drugs: 2013 update. Ann Rheum Dis 2014;73:492-509.

8 Smolen JS, Emery P, Fleischmann R, et al. Adjustment of therapy in rheumatoid arthritis on the basis of achievement of stable low disease activity with adalimumab plus methotrexate or methotrexate alone: the randomised controlled OPTIMA trial. Lancet 2014;383:321-32.

9 Lukas C, van der Heijde D, Fatenajad S, et al. Repair of erosions occurs almost exclusively in damaged joints without swelling. Ann Rheum Dis 2010;69:851-5.

10 Schett G, Gravallese E. Bone erosion in rheumatoid arthritis: mechanisms, diagnosis and treatment. Nat Rev Rheumatol 2012;8:656-64.

11 Monti S, Montecucco C, Bugatti S, et al. Rheumatoid arthritis treatment: the earlier the better to prevent joint damage. RMD Open 2015;1(Suppl 1):e000057.

12 van Vollenhoven RF, Nagy G, Tak PP. Early start and stop of biologics: has the time come? BMC Med 2014;12:25.

13 Ochi T, Iwase R, Yonemasu K, et al. Natural course of joint destruction and fluctuation of serum $\mathrm{C} 1 \mathrm{q}$ levels in patients with rheumatoid arthritis. Arthritis Rheum 1988;31:37-43. 
14 Kyburz D, Gabay C, Michel BA, et al. The long-term impact of early treatment of rheumatoid arthritis on radiographic progression: a population-based cohort study. Rheumatology (Oxford) 2011;50:1106-10.

15 Raza K, Saber TP, Kvien TK, et al. Timing the therapeutic window of opportunity in early rheumatoid arthritis: proposal for definitions of disease duration in clinical trials. Ann Rheum Dis 2012;71:1921-3.

16 Smolen JS, Aletaha D. Rheumatoid arthritis therapy reappraisal: strategies, opportunities and challenges. Nat Rev Rheumatol 2015;11:276-89.

17 Hoff M, Kvien TK, Kälvesten J, et al. Adalimumab reduces hand bone loss in rheumatoid arthritis independent of clinical response: subanalysis of the PREMIER study. BMC Musculoskelet Disord 2011;12:54.

18 Detert J, Bastian H, Listing J, et al. Induction therapy with adalimumab plus methotrexate for 24 weeks followed by methotrexate monotherapy up to week 48 versus methotrexate therapy alone for DMARD-naive patients with early rheumatoid arthritis: HIT HARD, an investigator-initiated study. Ann Rheum Dis 2013;72:844-50.

19 Smolen JS, Breedveld FC, Burmester GR, et al. Treating rheumatoid arthritis to target: 2014 update of the recommendations of an international task force. Ann Rheum Dis 2016;75:3-15.

20 Zhou Z, Rahme E, Abrahamowicz M, et al. Survival bias associated with time-to-treatment initiation in drug effectiveness evaluation: a comparison of methods. Am J Epidemiol 2005;162:1016-23.

21 Singh JA, Saag KG, Bridges SL Jr, et al. 2015 American College of Rheumatology Guideline for the Treatment of Rheumatoid Arthritis. Arthritis Rheumatol 2016;68:1-26.
22 Bathon JM, Martin RW, Fleischmann RM, et al. A comparison of etanercept and methotrexate in patients with early rheumatoid arthritis. N Engl J Med 2000;343:1586-93.

23 Breedveld FC, Weisman MH, Kavanaugh AF, et al. The PREMIER study: a multicenter, randomized, double-blind clinical trial of combination therapy with adalimumab plus methotrexate versus methotrexate alone or adalimumab alone in patients with early, aggressive rheumatoid arthritis who had not had previous methotrexate treatment. Arthritis Rheum 2006;54: 26-37.

24 Kavanaugh A, Fleischmann RM, Emery $\mathrm{P}$, et al. Clinical, functional and radiographic consequences of achieving stable low disease activity and remission with adalimumab plus methotrexate or methotrexate alone in early rheumatoid arthritis: 26-week results from the randomised, controlled OPTIMA study. Ann Rheum Dis 2013;72:64-71.

25 St Clair EW, van der Heijde DM, Smolen JS, et al. Combination of infliximab and methotrexate therapy for early rheumatoid arthritis: a randomized, controlled trial. Arthritis Rheum 2004;50:3432-43.

26 Takeuchi T, Yamanaka H, Ishiguro N, et al. Adalimumab, a human anti-TNF monoclonal antibody, outcome study for the prevention of joint damage in Japanese patients with early rheumatoid arthritis: the HOPEFUL 1 study. Ann Rheum Dis 2014;73:536-43.

27 Takahashi C, Kaneko Y, Okano Y, et al. THU0118 Methotrexate Polyglutamates in Erythrocytes Correlates with Clinical Response in Japanese Patients with Rheumatoid Arthritis. Ann Rheum Dis 2014;73(Suppl 2):218-19. 